\title{
Adrenal Extramedullary Hematopoiesis: A Case Report
}

\section{Mohsen Esfandbod* and Maryam Kabootari}

Department of Hematology and Oncology, Tehran University Medical Sciences, Tehran, Iran

\begin{abstract}
An adrenal incidentaloma is a mass lesion greater than $1 \mathrm{~cm}$ in diameter, serendipitously discovered by radiologic examination. Extramedullary hematopoiesis presenting as adrenal mass is not a common entity but foci of Extramedullary Hematopoiesis (EMH) may occur in almost any organ.
\end{abstract}

Keywords: Extramedullary hematopoiesis; Adrenal incidentaloma

\section{Introduction}

Extramedullary hematopoiesis arises in organs which are provided of pluripotent stem cells. Most commonly it occurs in the spleen, liver and lymph nodes; less frequently it is detected in the lung, pleura, breast, thymus, small bowel and central nervous system [1]. Very rarely, extramedullary hematopoiesis is finding out in the kidneys as well as adrenals [2]. Adrenal incidentalomas presenting with hematologic disorders, such as agnogenic myeloid aplasia and beta-thalassaemia, need careful imaging as well as adrenal hormonal investigation in order to exclude malignancy and subclinical hypersecretory syndromes. Ultrasound or CT-FNA of the lesion is effective in finding out the disease [3]. Herein we report a rare case of adrenal extramedullary hematopoiesis which was clinically detected as incidentaloma.

\section{Case Report}

Evaluation of non-specific abdominal pain in 23-year-old man with a history of Beta thalassaemia major led to finding of an unexpected adrenal incidentaloma. The patient was a 23 -year-old man who was diagnosed with Beta Thalassaemia major since four months of age and was under treatment with regular blood transfusions.

About 2 weeks before presentation during abdominal sonography, for evaluation of rise in liver enzymes, a large right retroperitoneal mass most probably adrenal in origin was detected. He denied any other symptoms such as abdominal pain, change in body weight, headache, palpitation, flushing, skin changes, weakness, hypertension or urinary tract symptoms.

A medical history was also significant for HCV infection since 14 years ago. He had received 1 year treatment with INF.

The patient's mother had a history of Diabetes mellitus. One cousin was also diagnosed with beta Thalassaemia major. Medications included Desferal, Folic acid and Vitamin C. The patient had no history of cigarette smoking or alcoholism.

For evaluation and diagnosis he was admitted in hospital. In physical examination vital signs were stable. Positive findings included Thalassaemic feature, pale conjunctivae, skin hyper pigmentation, systolic murmur III/VI in apex and lower sternal border, S3 gallop, asymmetric, mild right side bulging in the abdomen, soft Hepatomegaly ( $3 \mathrm{~cm}$ under right costal margin), splenomegaly ( $4 \mathrm{~cm}$ under left costal margin) and a large mass in RUQ and RLQ without tenderness. There was no lymphadenopathy. Extremity and neurologic examination was normal. Laboratory results revealed anemia with low MCV, increased AST, ALT and serum ferritin level.

In abdominal sonography liver span was $15 \mathrm{~cm}$ with coarse echo. There was also Splenomegaly (span: $16 \mathrm{~cm}$ ) and a large heterogeneous mass in right adrenal region. There was no ascites or lymphadenopathy. Abdominal spiral CT scan with oral and IV contrast showed a $10 \times 12$ $\mathrm{cm}$ mass in the right adrenal gland with calcified and necrotic zones with regular border. The mass displaced the liver and right kidney (Figure 1). In laboratory evaluation urine free cortisol, overnight dexamethasone suppression test, serum ACTH, Urine $24 \mathrm{hr}$ VMA, Urine $24 \mathrm{hr}$ Metanephrine and normetanephrine, serum DHEAS, aldosterone, renin, 17-hydroxyprogrstrone were all in the normal range.

Shortly after hospitalization surgical laparotomy and excision of the right adrenal gland was performed. Wedge biopsy was also taken from the liver. Histopathologic evaluation revealed an encapsulated irregular soft mass $13 \times 11 \times 5 \mathrm{~cm}$ weighing 379 gram. Cut surface was fragile and homogenous. Sections of the adrenal mass showed extensive proliferation of trilineage hematopoietic elements including megakaryocytes that were compatible with the diagnosis of extramedullary hematopoiesis. (Figure 2) Histological evaluation of the liver showed architecturally preserved liver tissue with mild portal inflammation, mild portal fibrosis and abundant golden brown pigment in the periportal hepatocytes. Our patient's postoperative course was uneventful, and he resumed normal oral intake. Resection even in asymptomatic patients has been recommended for patients with adrenal masses greater than $4 \mathrm{~cm}$ in diameter. Postoperative outcome is generally favorable, and recurrence after complete resection is unusual. We suggested a repeat imaging study at 6 to 12 months after

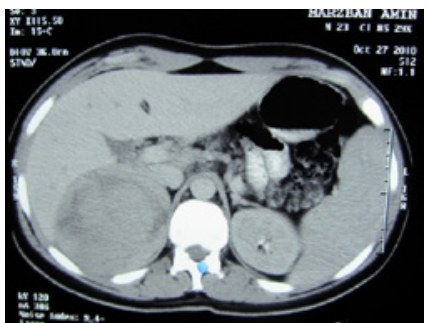

Figure 1: Non-contrast axial CT image of the abdomen, demonstrating a heterogeneous solid right suprarenal mass and an enlarged spleen.

*Corresponding author: Mohsen Esfandbod, Department of Hematology and Oncology, Tehran University Medical Sciences, Tehran, Iran, E-mail: sfandbod@sina.tums.ac.ir

Received July 26, 2012; Accepted November 19, 2012; Published November 21, 2012

Citation: Esfandbod M, Kabootari M (2012)Adrenal Extramedullary Hematopoiesis: A Case Report. J Clin Case Rep 2:226. doi:10.4172/2165-7920.1000226

Copyright: (C) 2012 Esfandbod M, et al. This is an open-access article distributed under the terms of the Creative Commons Attribution License, which permits unrestricted use, distribution, and reproduction in any medium, provided the original author and source are credited. 


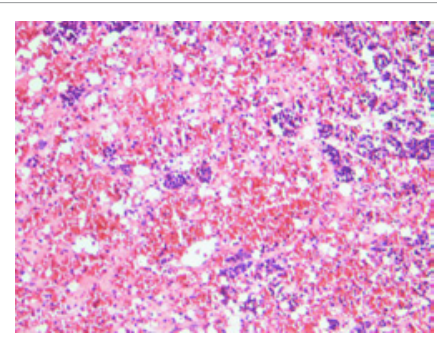

Figure 2: Smear shows megakaryocytes, nucleated red blood cell, and myelocytes. (May-Grunwald-Giemsa, X1200).

surgical resection. Strict chelating therapy for the iron overload state was also recommended.

\section{Discussion}

Hematopoiesis occurring at the sites other than the medullary cavity of bone is known as Extramedullary Hematopoiesis (EMH). It occurs when there is increased destruction of Red Blood Cells (RBC) as in hemolytic anemia and myelofibrosis [2]. An adrenal incidentaloma is a mass lesion greater than $1 \mathrm{~cm}$ in diameter, serendipitously discovered by radiologic examination [3]. Extramedullary hematopoiesis presenting as adrenal mass is not a common entity but foci of Extramedullary Hematopoiesis (EMH) may occur in almost any organ. this phenomenon can accompany a number of congenital hemolytic anemias or chronic myeloproliferative disease including Thalassaemia, sickle cell anemia, polycythemia rubra vera, chronic myelogenous leukemia, agnogenic myeloid metaplasia and hereditary spherocytosis. Organ involvement may present as splenomegaly, hepatomegaly, lymphadenopathy, pleural, pericardial or abdominal effusions or involvement of the gastrointestinal or genitourinary tracts or lung, leading to symptoms such as dysuria and respiratory distress. Involvement of the central nervous system may be associated with increased intracranial pressure, altered sensorium, motor and sensory impairment, including cord compression. Its identification is invariably incidental, imaging of the lesion is poorly specific and the diagnosis requires the histological examination [4].

\section{Conclusion}

In our review of literature we found reports of adrenal gland in patients with Beta-Thalassaemia, Agnogenic Myeloid Metaplasia and hereditary spherocytosis [1-7]. Surgery or radiotherapy should be performed only when EMH tumors cause symptoms, such as during spinal cord compression. Adrenal EMH tumors should be considered in Thalassaemia patients with an adrenal mass, to avoid unnecessary surgical procedures [7].

\section{References}

1. Porcaro AB, Novella G, Antoniolli SZ, Martignoni G, Brunelli M, et al. (2001) Adrenal extramedullary hematopoiesis: Report on a pediatric case and update of the literature. International Urology and Nephrology 33: 601-603.

2. Ahuja S, Grover G, Jha AK, Sodhi K, Bansal D, et al. (2011) Extramedullary Hematopoiesis presented as Solitary Renal Mass: A Case Report With Review of Literature. Diagn Cytopathol 6: 435-437.

3. Salemme M, Rodella R, Fisogni S, Facchetti F (2011) Nodular extramedullary hematopoiesis involving the adrenal gland. An uncommon cause of adrenal "incidentaloma". Pathologica 103: 46-49.

4. Calhoun SK, Murphy RC, Shariati N, Jacir N, Bergman K (2001) Extramedullary Hematopoiesis in a child with hereditary Spherocytosis: an uncommon cause of an adrenal mass. Pediatr Radiol 31: 879-881.

5. Keikhaei B, Soltani Shirazi A, Mohammad Pour M (2012)Adrenal extramedullary hematopoiesis associated with $\beta$-Thalassemia major. Hematol Rep 4: e7.

6. Chuang CK, Chu SH, Fang JT, Wu JH (1998) Adrenal extramedullary hematopoietic tumor in a patient with beta-thalassemia. J Formos Med Assoc 97: 431-433.

7. Young WF Jr (2007) Clinical practice. The incidentally discovered adrenal mass. N Engl J Med 356: 601-610. 